\title{
Block Principal Component Analysis for Extraction of Informative Features for Classification of Hyperspectral Images
}

\author{
Igor A. Pestunov and Pavel V. Melnikov* \\ Institute of Computational Technologies of SB RAS \\ 6 Akademika Lavrenteva, Novosibirsk, 630090, Russia
}

Received 04.02.2015, received in revised form 30.03.2015, accepted 20.05.2015

This paper proposes a method to reduce the dimensionality of feature space for recognition of hyperspectral images. The method consists of dividing the spectral channels into blocks with high in-block correlation and the subsequent application of principal component analysis. It is shown that the proposed method allows to reduce the number of channels used in the classification by an order of magnitude with no significant degradation of recognition quality.

Keywords: hyperspectral image, informative feature extraction, principal component analysis, supervised classification, support vector machine.

DOI: 10.17516/1999-494X-2015-8-6-715-725.

\section{Блочный метод главных компонент}

\section{для выделения информативных признаков}

при классификации гиперспектральных изображений

\author{
И.А. Пестунов, П.В. Мельников
}

Институт вычислительных технологий СО РАН Россия, 630090, Новосибирск, пр. Лаврентьева, 6

В статье предложен метод снижения размерности пространства признаков при распознавании гиперспектральных изображений, заключающийся в разбиении спектральных каналов на блоки с высокой коррелящией с последующим применением метода главных компонент. Показано, что предлагаемый метод позволяет на порядок сократить число используемых при классификачии спектральных признаков без значительного ухудшения качества распознавания.

Ключевые слова: гиперспектральное изображение, выделение информативных признаков, метод главных компонент, обучаемая классификация, метод опорных векторов.

(C) Siberian Federal University. All rights reserved

* Corresponding author E-mail address: pestunov@ict.nsc.ru,pvlvlml@gmail.com 


\section{Введение}

В настоящее время в связи с интенсивным развитием средств и технологий дистанционного зондирования все большую актуальность приобретает задача распознавания гиперспектральных изображений [1]. Главными особенностями таких изображений является большое число спектральных каналов, которое может достигать нескольких сотен, и малая ширина каждого канала (порядка нескольких нанометров). Гиперспектральные изображения - фактически трехмерные массивы данных, в которых два измерения соответствуют пространственным координатам, а третье - спектральной координате изображения, поэтому гиперспектральные изображения также называют гиперкубами [2].

Значительное увеличение числа каналов в гиперспектральном изображении приводит к невозможности применения большинства традиционных алгоритмов обработки мультиспектральных изображений. Ограничения могут быть связаны как с теоретическими требованиями их корректного применения к данным большой размерности, так и с неприемлемым увеличением времени работы или объема используемой памяти.

При классификации гиперспектральных изображений с обучением актуальной становится проблема репрезентативности обучающей выборки. Известно [3], что для обеспечения приемлемого качества классификации минимальное количество точек обучающей выборки для параметрических классификаторов составляет $10 k$ точек на каждый класс (где $k$ - число каналов), для непараметрических - $50 k$ точек. На практике получение обучающих выборок таких объемов, как правило, не представляется возможным.

Одним из подходов к решению данных проблем служит разработка новых эффективных алгоритмов классификации, учитывающих особенности гиперспектральных изображений [4, 5]. Другим подходом является выбор и выделение информативных подсистем признаков, которые позволят использовать традиционные алгоритмы, хорошо зарекомендовавшие себя при обработке мультиспектральных изображений [6]. При большом числе каналов и высоком спектральном разрешении соседние каналы зачастую обладают значительной взаимной корреляцией и использование всех каналов при анализе изображения может оказаться нецелесообразным. Для сокращения числа признаков применяют два основных подхода: выбор признаков из множества каналов исходного изображения [7] и получение новых признаков путем линейных или нелинейных преобразований исходных каналов изображения. Преобразование каналов выполняется с помощью различных методов, например метода главных компонент [8], факторного анализа [9], вейвлет-преобразований [10] и др.

В настоящей статье предлагается процедура генерации минимальных систем информативных признаков при распознавании гиперспектральных изображений, заключающаяся в разбиении каналов изображения на блоки с высокой внутригрупповой корреляцией с последующим применением метода главных компонент.

При разработке и исследовании данного метода в качестве тестового использовалось гиперспектральное изображение Indian Pines, полученное сенсором AVIRIS 12 июня 1992 г. [11]. Это изображение часто применяется при разработке и исследовании алгоритмов анализа гиперспектральных изображений [12]. Размер изображения составляет $145 \times 145$ пикселей, каждый пиксель представлен вектором из 224 значений спектральных яркостей в диапазоне 400-2500 нм. Для него имеется эталонная обучающая выборка из 16 тематических классов 


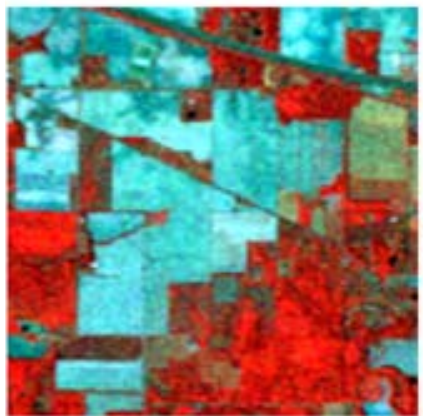

a

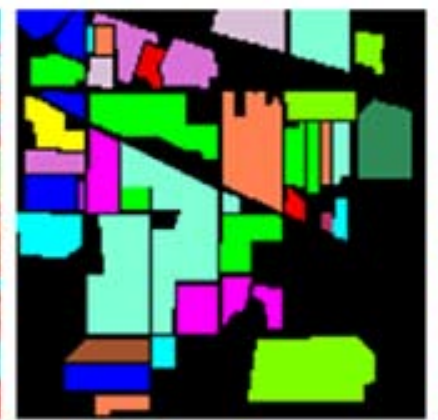

6

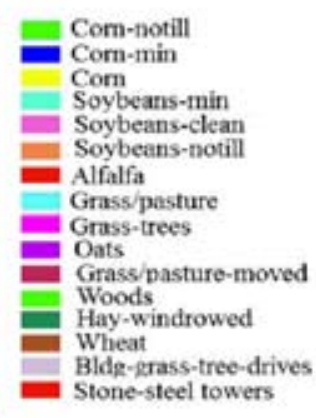

Stone-steel towers

Рис. 1. RGB-композит (а) и эталонная картосхема (б) тестового изображения Indian Pines

(посадки кукурузы, сои, люцерны, пшеницы и др.). RGB-композит исходного изображения (составленный с использованием каналов 54, 112 и 204) и эталонная картосхема изображены на рис. 1.

\section{Описание предлагаемого метода}

Анализ корреляционной матрицы исходного гиперспектрального изображения Indian Pines показывает, что сильно коррелированные спектральные каналы располагаются блоками. На рис. 2 матрица корреляции каналов данного изображения представлена в виде полутонового изображения, на котором яркость каждого пикселя отражает значение соответствующего элемента матрицы корреляции (темный цвет - сильная отрицательная корреляция, светлый сильная положительная, серый - слабая корреляция).

Для разбиения каналов изображения на блоки предлагается следующий пороговый алгоритм:

Шаг 1. Положить $B:=1$ - число каналов в текущем блоке, $b_{0}:=1$ - номер первого канала текущего блока.

Шаг 2. Для каждого канала $i=2, \ldots, k$ (где $k$ - число каналов на изображении):

Шаг 2.1. Рассчитать среднюю корреляцию канала со всеми каналами текущего блока: $\rho:=\frac{1}{B} \sum_{j=b_{0}}^{b_{0}+B-1}\left|r_{i, j}\right|$, где $\left[r_{i, j}\right]_{k \times k}-$ матрица корреляции.

Шаг 2.2. Если данный канал имеет сильную корреляцию с текущим блоком, т.е. $\rho>T$, то он добавляется в текущий блок $(B:=B+1)$, иначе происходит создание нового блока: пара $\left(b_{0}, B\right)$ добавляется в результирующий список блоков, $B:=1, b_{0}:=\mathrm{i}$ (здесь $T$ - заданный порог, который определяет необходимое для конкретной задачи число блоков).

Шаг 2.3. Перейти на шаг 2.

Проведенные эксперименты показали, что при выборе порога $T=0.95$ каналы изображения разбиваются на пять блоков, которые обладают высокой внутригрупповой корреляцией ( $\rho>0.95)$, диапазоны спектра, охватываемые этими блоками, приблизительно соответствуют видимому, ближнему и среднему инфракрасному диапазонам спектра (дополнительно инфракрасные диапазоны оказались разбиты на несколько блоков). Визуальный анализ спектраль- 
ных кривых пикселей изображения (рис. 3) показывает, что каналы 99-110 и 148-166 обладают малой дисперсией и не содержат полезной информации. Это обусловлено тем, что в этих диапазонах спектра находятся полосы поглощения атмосферы Земли [12].

Разбиение каналов исходного изображения на пять блоков, полученное в результате применения приведенного алгоритма, представлено на рис. 4.

Таким образом, были выделены блоки спектральных каналов исходного изображения, внутри которых наблюдается сильная корреляция. Для уменьшения объема и снижения размерности данных к каждому блоку применяется метод главных компонент [13]. Этот метод предназначен для выделения некоррелированных линейных комбинаций признаков из коррелированных данных [14]. На следующем этапе из полученных главных компонент необходимо

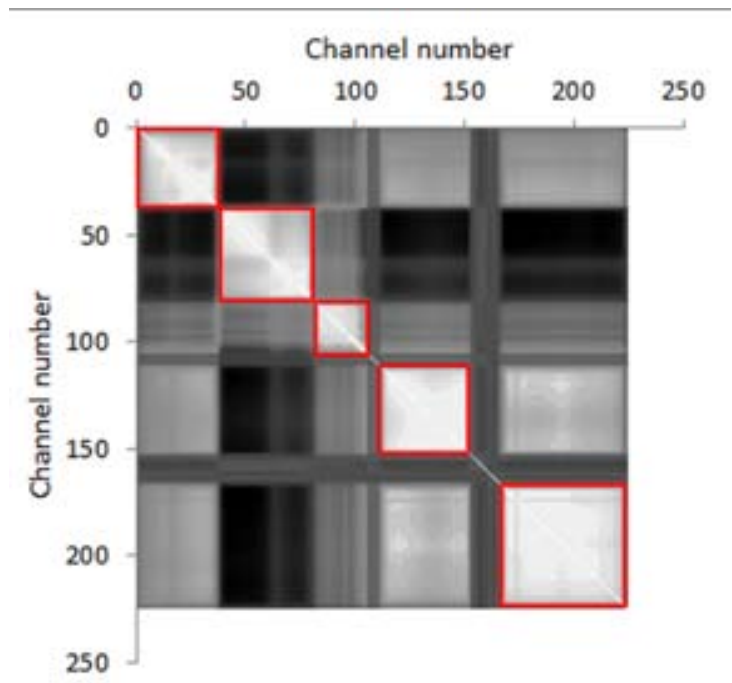

Рис. 2. Полутоновая визуализация корреляционной матрицы каналов тестового изображения (красным цветом выделены блоки каналов с сильной корреляцией)

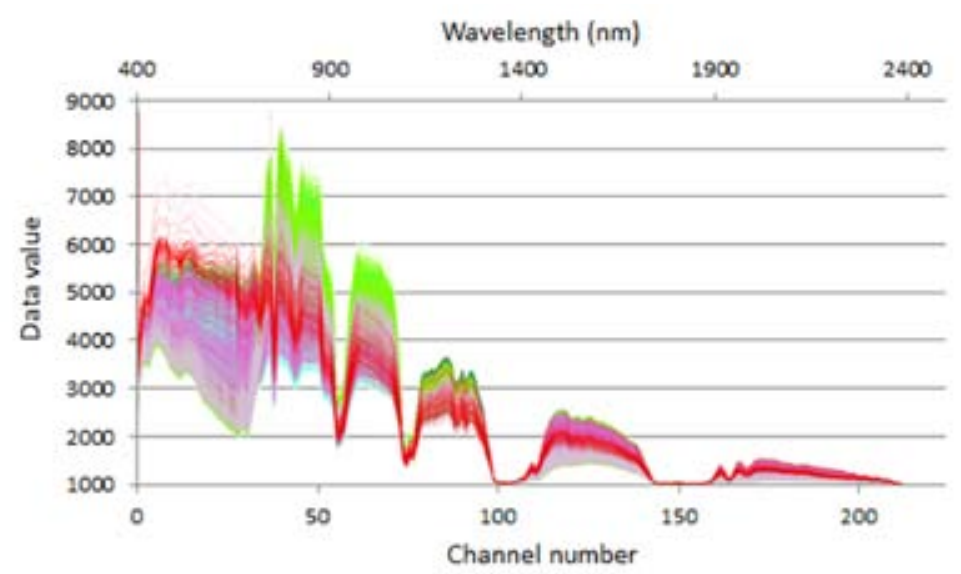

Рис. 3. Спектральные кривые пикселей эталонной картосхемы тестового изображения (цветом показана принадлежность пикселей к определенным классам) 


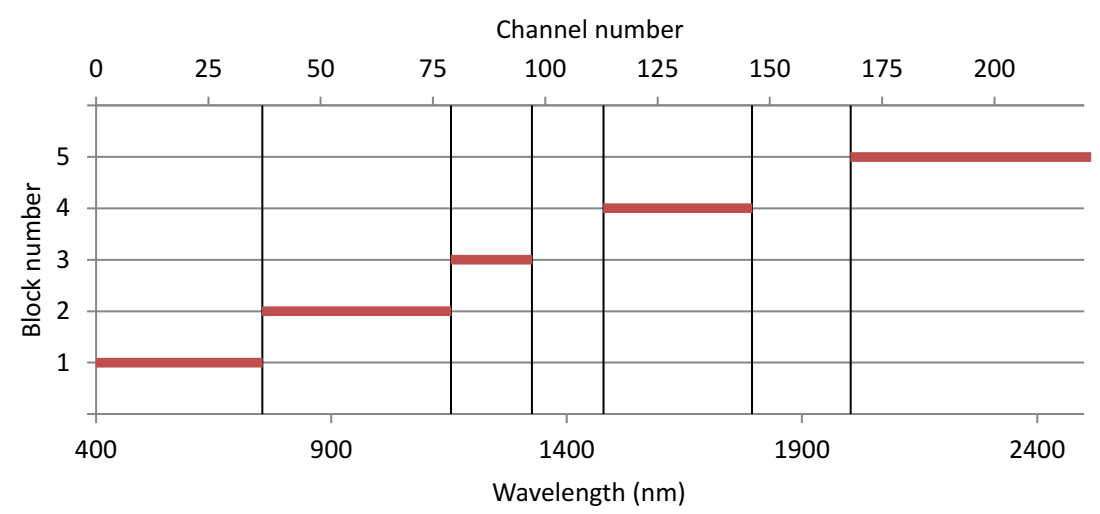

Рис. 4. Разбиение спектрального диапазона изображения на пять блоков (цветом выделены диапазоны, соответствующие каждому блоку)

выбрать информативные признаки (содержащие информацию об объектах на изображении). Сделать это можно различными способами:

1. Посредством визуального анализа полученных компонент внутри каждого блока. Этот способ позволяет относительно точно оценить информативность каждого признака и использовать для дальнейшей работы те признаки, которые позволяют эксперту выделить объекты интереса. Однако такой подход субъективен, он не может обеспечить повторяемость результатов.

2. Путем анализа дисперсии каждой компоненты или собственных значений ковариационной матрицы, полученных в результате применения метода главных компонент. Признаки, обладающие дисперсией больше заданного порога, считаются информативными и используются для дальнейшей работы. Исследование показало, что величина собственных чисел для главных компонент, которые визуально являются информативными, существенно отличаются для различных блоков признаков. Поэтому не представляется возможным установить одно общее пороговое значение для всех блоков признаков, величина порога для каждого блока должна выбираться «вручную». Таким образом, данный способ требует наличия такой же экспертной информации, что и первый. Он дает тот же результат, но более сложен в реализации.

3. Пропорционально количеству признаков в блоке, для которого вычисляются главные компоненты. Этот подход не всегда дает корректные результаты, так как блок может иметь значительное количество каналов, однако все они могут быть сильно коррелированными и обладать малым количеством информативных главных компонент.

Таким образом, из трех предлагаемых подходов корректные результаты обеспечивают первые два. При проведении экспериментов в данной работе использовался первый подход.

На рис. 5а представлены первые 36 каналов исходного изображения, составляющие первый блок, на рис. $5 б$ - вычисленные по этим каналам главные компоненты. Рисунки показывают, что для дальнейшей обработки можно использовать только первые четыре главных компоненты, так как остальные обладают слишком малой дисперсией и являются малоинформативными.

Предлагаемый алгоритм выделения подсистем информативных признаков (блочный метод главных компонент, БМГК) можно записать в виде последовательности шагов.

$$
-719-
$$




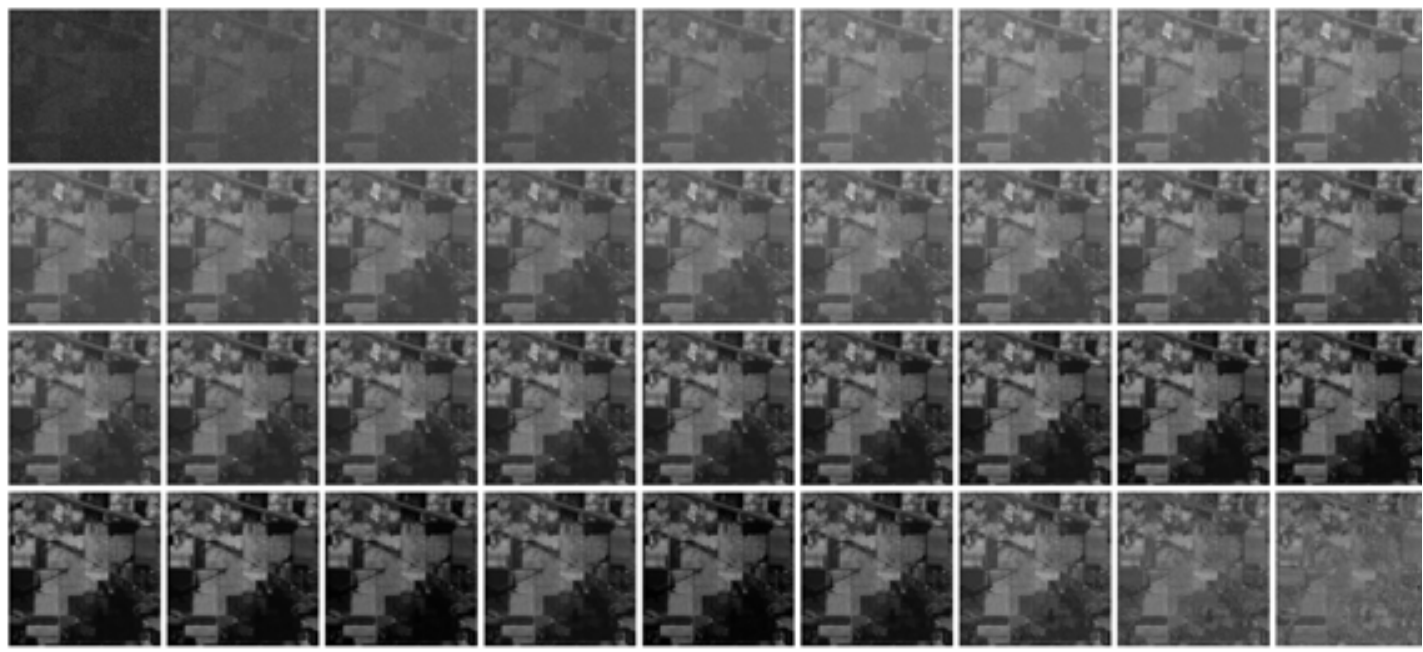

a

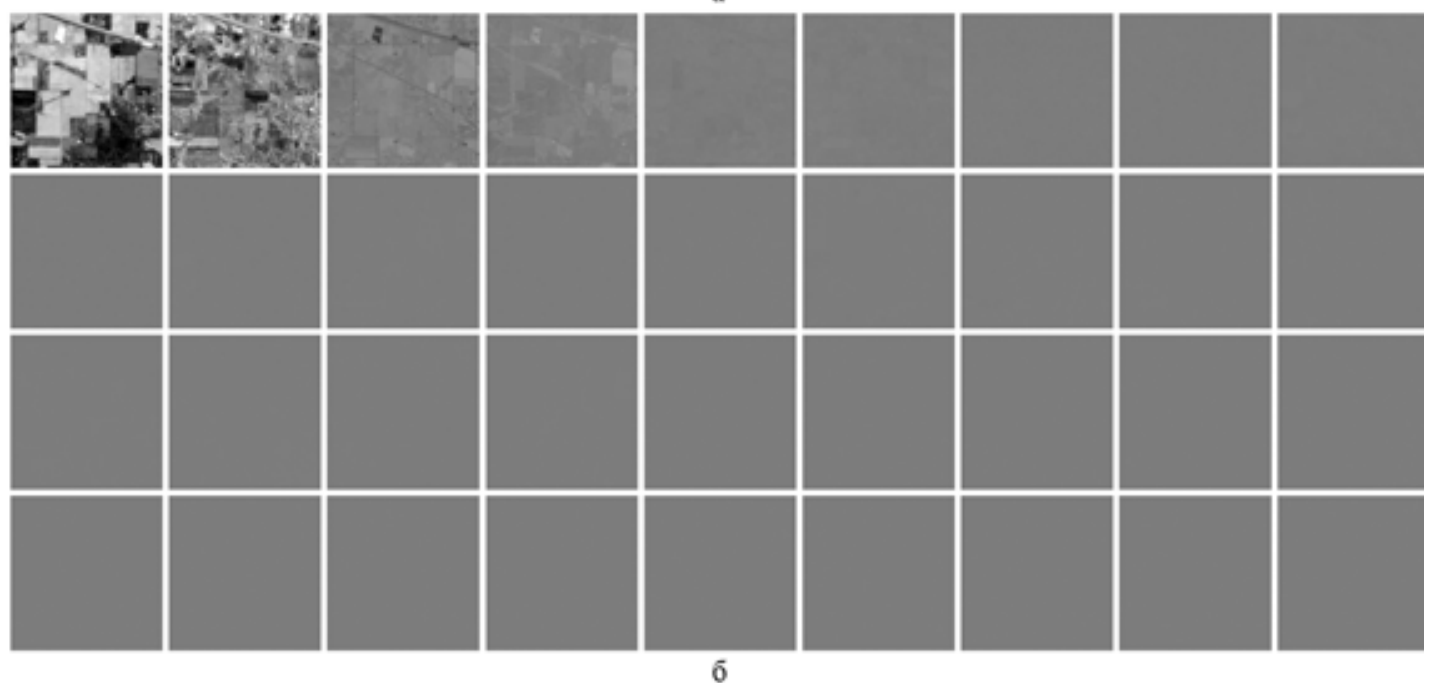

Рис. 5. Каналы 1-36 тестового изображения (а) и вычисленные по ним главные компоненты (б)

Шаг 1. Разделить множество каналов изображения на блоки на основе анализа матрицы корреляции с помощью предложенного порогового алгоритма.

Шаг 2. Вычислить главные компоненты для каждого блока каналов изображения.

Шаг 3. В каждом блоке выбрать информативные признаки посредством визуального анализа.

В соответствии с предлагаемым методом по 224 каналам исходного изображения был построен набор, состоящий из 21 информативного признака. В табл. 1 приведено число главных компонент, выбранное для каждого блока каналов.

\section{3. Результаты экспериментальных исследований}

Для оценки эффективности предложенного метода было выполнено сравнение результатов классификации тестового изображения по всем спектральным каналам и с использованием

$$
-720-
$$


Таблица 1. Число информативных компонент для каждого блока каналов

\begin{tabular}{|l|c|c|c|c|c|}
\hline \multicolumn{1}{|c|}{ Номер блока } & 1 & 2 & 3 & 4 & 5 \\
\hline Число исходных каналов & 36 & 42 & 19 & 34 & 57 \\
\hline Число главных компонент & 4 & 5 & 3 & 4 & 5 \\
\hline
\end{tabular}

различных подсистем признаков, включая набор признаков, полученных предложенным методом.

Для обучаемой классификации использовался метод опорных векторов (Support Vector Machine, SVM), который получил широкое распространение в последние годы $[15,16]$. SVM является одним из немногих алгоритмов, которые можно корректно применять для классификации данных высокой размерности [17]. Он реализован во многих пакетах обработки данных дистанционного зондирования (например, в пакете EXELIS ENVI [18]). В данном исследовании использовался алгоритм SVM с ядром на основе радиальной базисной функции (RBF).

Для классификации также использовался алгоритм, учитывающий пространственную информацию [19]. Он позволяет повышать качество картосхем, полученных попиксельными алгоритмами классификации (метод опорных векторов, метод минимального расстояния, метод максимального правдоподобия и др.) путем использования информации о соседстве пикселей (локальном контексте изображения). Для этого по изображению строится взвешенный граф, вершинами которого являются пиксели изображения, а ребра соединяют смежные пиксели. Вес ребра определяется с помощью некоторой функции похожести пикселей в пространстве спектральных признаков. Построенный граф служит источником пространственной информации для уточнения картосхемы, полученной в ходе попиксельной классификации. Вершины графа, соответствующие выбранным на картосхеме пикселям, помечаются метками классов этих пикселей, помеченные вершины соединяются виртуальными ребрами, после чего выполняется выделение минимального остовного дерева с использованием классического алгоритма Крускала [20]. Чтобы обеспечить независимость результата от выбора маркеров, применяется ансамблевый подход. На каждой итерации ансамбля выбирается случайный набор маркеров, итоговая сегментация изображения строится на основе коллективного решающего правила большинства.

Для оценки качества классификации была использована процедура скользящего экзамена, позволяющая получить несмещенную оценку вероятности ошибки классификации [21], а также процедура оценки на контрольной выборке с применением обучающих выборок различного размера.

Процедура скользящего экзамена проводилась с использованием метода опорных векторов по шести наборам признаков:

1) все нешумовые каналы тестового изображения (188 признаков),

2) 21 признак, полученный с помощью предлагаемого метода,

3) 21 главная компонента, вычисленная традиционным методом с использованием всех 188 нешумовых каналов,

4) набор из 5 признаков (первые главные компоненты каждого блока), 
Таблица 2. Точность классификации при использовании различных наборов признаков

\begin{tabular}{|l|c|c|}
\hline \multicolumn{1}{|c|}{ Набор признаков } & $\begin{array}{c}\text { Количество } \\
\text { признаков }\end{array}$ & $\begin{array}{c}\text { Точность } \\
\text { классификации, \% }\end{array}$ \\
\hline Все исходные признаки & 188 & 92.04 \\
\hline Признаки, построенные с помощью блочного МГК & 21 & 91.11 \\
\hline 21 главная компонента, вычисленная традиционным МГК & 21 & 75.48 \\
\hline По одной главной компоненте из каждого блока & 5 & 84.60 \\
\hline По две главных компоненты из каждого блока & 10 & 88.19 \\
\hline По три главных компоненты из каждого блока & 15 & \\
\hline
\end{tabular}

5) набор из 10 признаков (первые две главные компоненты каждого блока),

6) набор из 15 признаков (первые три главные компоненты каждого блока).

Результаты классификации по каждому из этих наборов данных приведены в табл. 2 (показаны результаты, усредненные по 15 испытаниям). Анализ таблицы показывает, что предложенный метод позволяет существенно сократить число используемых признаков при незначительном ухудшении качества классификации. Из таблицы также видно, что традиционный способ применения метода главных компонент (без разбиения всех каналов на коррелированные блоки) уступает по эффективности предлагаемому методу.

Второй эксперимент проводился для оценки качества работы алгоритма в условиях, близких к реальным. В ходе эксперимента был осуществлен методом оценки на контрольной выборке с использованием обучающих выборок объемом 25, 50, 100, 200, 300 и 400 точек на класс. Точки обучающей выборки выбирались на эталонной картосхеме случайным образом. Для классов, содержащих меньше точек, чем необходимый объем обучающей выборки, выбиралось по 15 точек. Производилось сравнение трех наборов признаков: 21 признак, полученный блочным методом главных компонент (БМГК), 21 главная компонента, полученная традиционным МГК, и полный набор из 188 каналов.

В данном эксперименте классификация проводилась с помощью двух алгоритмов:

1) метод опорных векторов (SVM),

2) метод классификации, построенный на основе SVM и учитывающий локальный контекст изображения (SVM+LC).

Результаты приведены на рис. 6. Из графика видно, что точность классификации по набору признаков БМГК в большинстве случаев выше, чем по полному набору признаков (особенно при малых объемах обучающей выборки). Набор из главных компонент, полученных без применения блочного подхода, дает худшее качество, чем полный и предлагаемый наборы признаков. На рис. 7 представлены результаты классификации по различным наборам признаков и при различных объемах обучающих выборок.

\section{Заключение}

В статье предложен блочный метод главных компонент (блочный МГК) для выделения информативного набора признаков в задачах, связанных с распознаванием гиперспектральных изображений. Блочный МГК заключается в применении метода главных компонент к блокам 


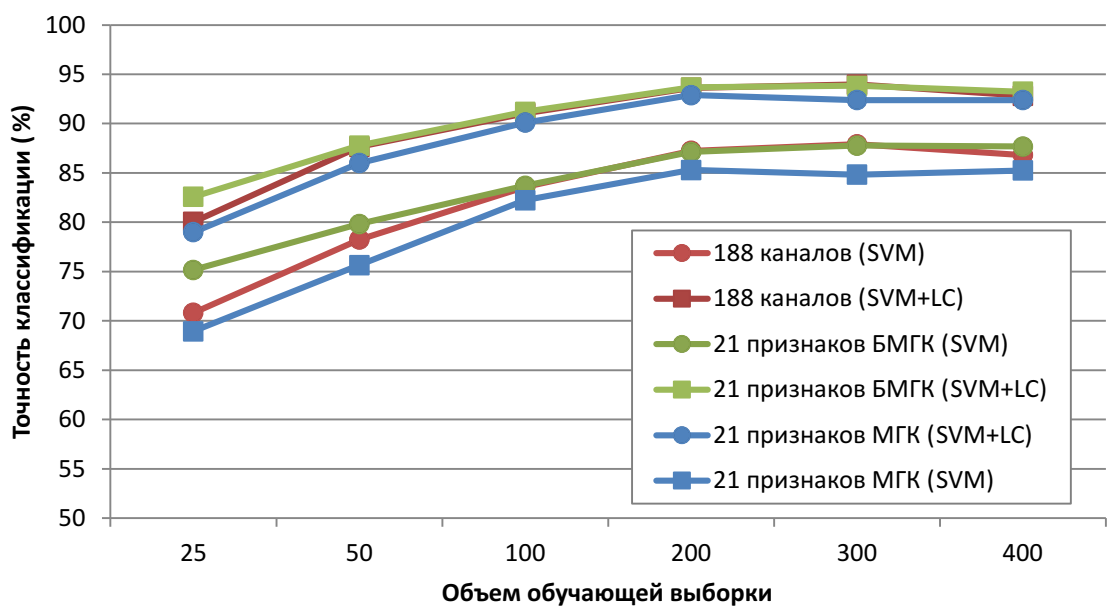

Рис. 6. Точность классификации при различных объемах обучающей выборки

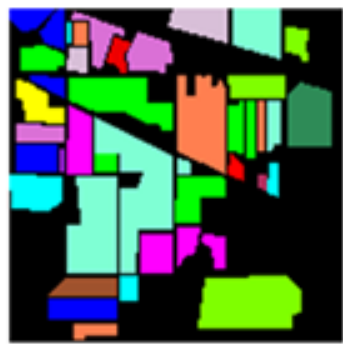

a

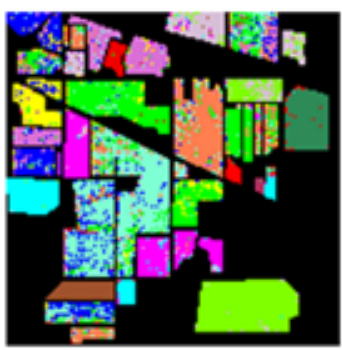

6

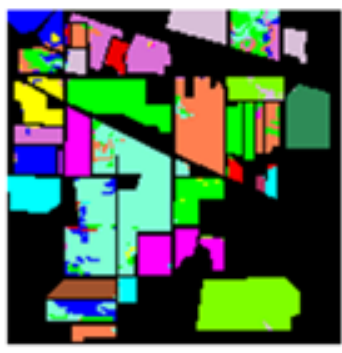

e

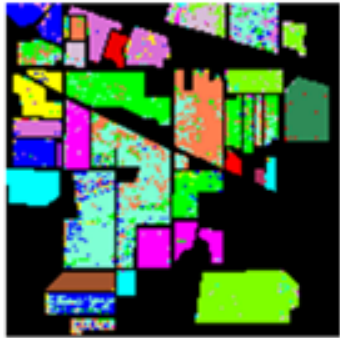

B

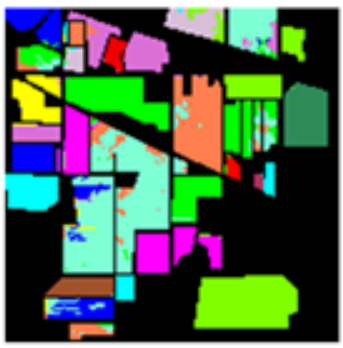

ж

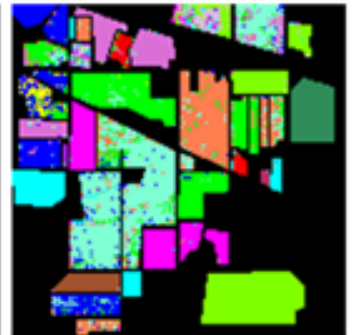

Г

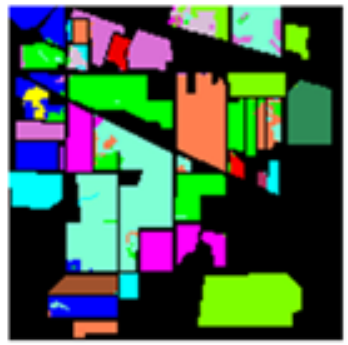

3

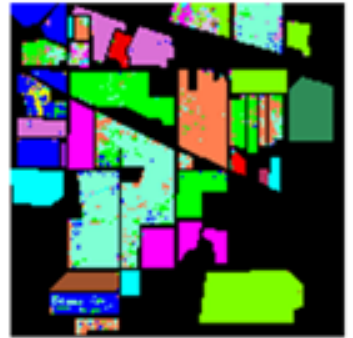

д

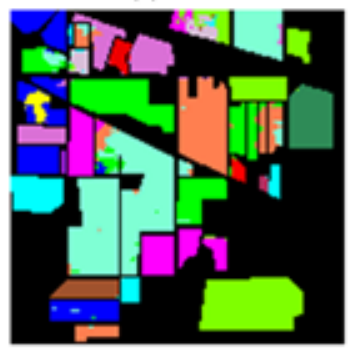

II

Рис. 7. Эталонная картосхема (а) и результаты классификации по различным наборам признаков: SVM: 50 точек OB (б - МГК, в - БМГК), 400 точек OB (г - МГК, д - БMГК), SVM+LC: 50 точек OB (е - MГК, ж- БМГК), 400 точек ОВ (з-МГК, и - БМГК) 
коррелированных каналов для выбора информативных компонент из каждого блока. Алгоритм позволяет на порядок сократить число используемых при распознавании признаков без существенного ухудшения качества классификации.

Показана эффективность предложенного метода в задачах обучаемой классификации на примере распознавания гиперспектрального изображения Indian Pines, полученного сенсором AVIRIS. Для оценки эффективности проводилась попиксельная классификация, основанная на методе опорных векторов, и контекстная классификация с использованием графового метода.

Работа выполнена при финансовой поддержке РФФИ (гранты № 13-07-12202-офи_м, № 14-07-31320-мол-а).

\section{Список литературы}

[1] Бондур В.Г. // Исследование Земли из космоса. 2014. № 1. С. 4-16.

[2] Borengasser M. Hyperspectral Remote Sensing - Principles and Applications. CRC Press, 2004. $128 \mathrm{p}$.

[3] Раудис P. // Статистические проблемы управления. Вильнюс: Институт математики и кибернетики АН ЛитССР, 1984. Вып. 66. С. 9-42.

[4] Antonio Plaza, Jon Atli Benediktsson, Joseph W. Boardman et. al. // Remote sensing of environment. 2009. Vol. 113. S. 110-122.

[5] Ablin R.C. Helen Sulochana. // International Journal of Advanced Research in Computer and Communication Engineering. 2013. Vol. 2. Is. 8. P. S2986-S3003.

[6] Louis Du Plessis, Rui Xu, Steven Damelin et. al. // International Journal of Systems, Control and Communications 3.3. 2011. P. 232-251.

[7] Martinez-Uso A., Pla F. Sotoca, J.M. et al. // IEEE Transactions on Geoscience and Remote Sensing Vol. 45.12. 2007. Р. 4158-4171.

[8] Остриков В.Н., Смирнов С.И., Михайлов В.В. // Современные проблемы дистанционного зондирования Земли из космоса: Тезисы докладов Двенадцатой Всероссийской открытой конференции. Москва: ИКИ РАН, 2014. С. 68 [http://smiswww.iki.rssi.ru/d33_conf/thesisshow. asp $x$ ?page $=91 \&$ thesis $=4482]$.

[9] Lavanya A., Sanjeevi S. // Journal of the Indian Society of Remote Sensing. 2013. Vol. 41.2. P. 199-211.

[10] Bruce L.M., Koger C.H., Jiang L. // IEEE Transactions on Geoscience and Remote Sensing. 2002. Vol. 40. P. 2331-2338.

[11] Porter W.M. Enmark H.E. // SPIE Proc. 1987. Vol. 834. P. 22-31.

[12] Hyperspectral Remote Sensing Scenes [Электронный ресурc]. URL: http://www.ehu.es/ ccwintco/index.php/Hyperspectral_Remote_Sensing_Scenes (дата обращения: 22.06.2014).

[13] Многозональные аэрокосмические съемки Земли / ред. Р.З. Сагдеев. М.: Наука, 1981. $303 \mathrm{c}$.

[14] Красиков В.А. и др. // Аэрокосмические исследования Земли. Обработка видеоинформации на ЭВМ / ред. В.Г. Золотухин. М.: Наука, 1978. С. 143-150.

[15] Cristianini N., Shawe-Taylor J. An Introduction to Support Vector Machines and other kernelbased learning methods. Cambridge University Press, 2000. ISBN 0-521-78019-5. 198 p. 
[16] LIBSVM - A Library for Support Vector Machines [Электронный ресурc]. URL: http:// www.csie.ntu.edu.tw/ cjlin/libsvm/ (дата обращения: 22.06.2014).

[17] Bernard Kévin et al. // IEEE Transactions on Image Processing. 2012. Vol. 21.4. P. 20082021.

[18] Support Vector Machine [Электронный ресурc]. URL: http://www.exelisvis.com/docs/ SupportVectorMachine.html.

[19] Пестунов И.А., Мельников П.В., Дубровская О.А. и др. // Интерэкспо ГЕО-Сибирь-2014: $\mathrm{X}$ Междунар. науч. конгр., 8-18 апреля 2014 г., Новосибирск: Междунар. науч. конф. «Экономическое развитие Сибири и Дальнего Востока. Экономика природопользования, землеустройство, лесоустройство, управление недвижимостью»: сб. материалов в 2 т. Т. 2. Новосибирск: СГГА, 2014. С. 357-366.

[20] Joseph. B. Kruskal // Proc. AMS. 1956. Vol. 7, N. 1. P. 48-50.

[21] Fukunaga K. Introduction to Statistical Pattern Recognition. 2nd Ed. N.Y.: Academic Press, 1990. $590 \mathrm{p}$. 